\title{
Terapia nutricionais nas doenças inflamatórias intestinais: Doença de Crohn e
}

\section{Retocolite Ulcerativa}

\author{
Nutritional therapy for inflammatory bowel diseases: Crohn's disease and ulcerative colitis \\ Terapia nutricional para enfermedades inflamatorias del intestino: enfermedad de Crohn y colitis \\ ulcerosa
}

Recebido: 28/05/2021 | Revisado:01/06/2021 | Aceito: 01/06/2021 | Publicado: 11/06/2021

\author{
Amanda Luizy Camara Santos \\ ORCID: https://orcid.org/0000-0002-7137-6721 \\ Centro Universitário FAMETRO, Brasil \\ E-mail: amandaluizy@ hotmail.com \\ Bianca Christina de Oliveira Dias \\ ORCID: https://orcid.org/0000-0001-7955-1144 \\ Centro Universitário FAMETRO, Brasil \\ E-mail: byanca_christina@outlook.com \\ Klines Alves da Silva \\ ORCID: https://orcid.org/0000-0001-6172-9979 \\ Centro Universitário FAMETRO, Brasil \\ E-mail: klinesalves_@hotmail.com \\ José Carlos de Sales Ferreira \\ ORCID: https://orcid.org/0000-0002-1867-8229 \\ Centro Universitário FAMETRO, Brasil \\ E-mail: jcarlos.sales@gmail.com
}

\begin{abstract}
Resumo
A terapia nutricional nas doenças inflamatórias intestinais vem se consolidando ao longo do tempo como um tratamento bastante eficaz e que tem como base uma dieta que vise combater essas patologias. Os meios nutricionais empregados obedecem aos critérios bem estruturados de acordo com os conhecimentos científicos adquiridos no extenso histórico de pesquisa desses aspectos da saúde dos indivíduos. O presente artigo ao tentar esclarecer esses benefícios, parte do pressuposto de que a incidência dessas enfermidades está altíssima e que para o seu devido tratamento, faz-se necessário uma gama maior de metodologias, dentre as quais destaca-se a terapia nutricional. Nas doenças inflamatórias crônicas as doenças inflamatórias intestinais estão classificadas como as de causas desconhecidas. Acredita-se que surjam da interação de quatro fatores fundamentais: ambiental, genético microbiano e imunológico. Essas doenças causam um grande impacto sobre a qualidade de vida do paciente, que é marcada por uma sobrecarga de terapias, hospitalizações e cirurgias.
\end{abstract}

Palavras-chave: Terapia nutricional; Doenças inflamatórias; Hospitalizações; Cirurgias.

\begin{abstract}
Nutritional therapy in inflammatory bowel diseases has been consolidated over time as a very effective treatment and based on a diet aimed at tackling these pathologies. The nutritional means used obey well-structured criteria according to the scientific knowledge acquired in the extensive research history of these aspects of the health of ours. The present article, when trying to clarify these benefits, starts from the assumption that the incidence of these diseases is very high and that for their due treatment, a greater range of methodologies is necessary, highlighting which ones stand out as a nutritional therapy. Chronic inflammatory diseases such as inflammatory bowel diseases are classified as of unknown causes. It is believed that they arise from the interaction of four fundamental factors: environmental, genetic, microbial and immunological. These diseases have a great impact on the patient's quality of life, which is marked by an overload of therapies, hospitalizations and surgeries. Keywords: Nutritional therapy; inflammatory diseases; hospitalizations; surgeries.
\end{abstract}

Keywords: Nutritional therapy; Inflammatory diseases; Hospitalizations; Surgeries.

\section{Resumen}

La terapia nutricional en las enfermedades inflamatorias intestinales se ha ido consolidando con el tiempo como un tratamiento muy eficaz y basado en una dieta que tiene como objetivo combatir estas patologías. Los medios nutricionales utilizados obedecen a criterios bien estructurados de acuerdo con los conocimientos científicos adquiridos en la extensa historia de investigación de estos aspectos de la salud de los individuos. Este artículo intenta 
esclarecer estos beneficios, partiendo del supuesto de que la incidencia de estas enfermedades es muy alta y que para su adecuado tratamiento es necesaria una mayor variedad de metodologías, entre las que destaca la terapia nutricional. En las enfermedades inflamatorias crónicas, las enfermedades inflamatorias del intestino se clasifican como las de causa desconocida. Se cree que surgen de la interacción de cuatro factores fundamentales: ambientales, genéticos, microbianos e inmunológicos. Estas enfermedades tienen un gran impacto en la calidad de vida del paciente, que está marcada por una sobrecarga de terapias, hospitalizaciones y cirugías.

Palabras clave: Terapia nutricional; Enfermedades inflamatorias; Hospitalizaciones; Cirugías.

\section{Introdução}

Nas doenças inflamatórias crônicas, as doenças inflamatórias intestinais estão classificadas como as de causas desconhecidas. Acredita-se que surjam da interação de quatro fatores fundamentais: ambiental, genético microbiano e imunológico. São doenças crônicas caracterizadas por desordens gastrointestinais e extra-intestinais e inclui dois tipos principais de enfermidade, a Doença de Crohn e a Retocolite Ulcerativa (Molodecky, et al., 2012).

As incidências de doenças inflamatórias intestinais tiveram um aumento e está relacionado principalmente com o desenvolvimento social e econômico da população e a ocidentalização do estilo de vida. Os países com alta incidência são os estados Unidos da América, Inglaterra, Itália Escandinávia e países do norte da Europa. Países do Sul da Europa África do Sul, Austrália e Nova Zelândia são países de incidência intermediária. Os de baixa incidência são: países da Ásia e da América do Sul.

A doença de Crohn é uma doença inflamatória crônica do trato gastrointestinal, seus sintomas evoluem de forma recorrente e remitente, causa danos ao intestino e incapacidade. Os pacientes apresentam um fenótipo inflamatório no momento do diagnostico, com o tempo as complicações se desenvolvem em metade dos pacientes, podendo resultar em cirurgia. (Thia, et al., 2010).

A doença traz um grande impacto sobre a qualidade de vida do paciente, que é marcada por uma sobrecarga de terapias, hospitalizações e cirurgias. Se dá de forma segmentar, é caracterizada por possuir segmentos saudáveis que separam os segmentos inflamados. (Beyer, et al., 2010). Algumas complicações da doença são fibrose intestinal, estenoses e formação de fistula (Iskandra, et al., 2012).

A retocolite ulcerativa é uma doença inflamatória crônica do cólon e reto que geralmente se apresenta com sangramento retal, diarreia, tenesmo e, às vezes, dor abdominal baixa. Os picos de incidência ocorrem entre 15 e 45 anos. Embora a causa exata da retocolite ulcerativa continue a ser elucidada, tornou-se claro na última década que a doença resulta de uma resposta imune intestinal desregulada impulsionada por uma interação complexa entre o genótipo do hospedeiro e a microbiota intraluminal ou outros patógenos potencialmente tóxicos (Souza, et al., 2016).

Os sintomas mais comuns da doença são diarreia e sangue nas fezes, dependendo da gravidade e da localização os pacientes também podem ter vários graus de dor abdominal, secreção de muco. Em casos raros com inflamação grave, os pacientes podem apresentar perda de peso, febre ou perfuração. Os sintomas começam gradualmente e evoluem ao longo de várias semanas. (Danese, et al., 2011).

A inflamação do trato gastrointestinal (TGI) interfere diretamente na qualidade de vida e no estado nutricional do indivíduo com doenças inflamatórias intestinais (DII). A alimentação pode estar envolvida na indução da inflamação, bem como em sua remissão (Hou, et al., 2011). Alguns alimentos ou a exclusão de alguns deles podem modular a resposta do TGI à inflamação, favorecendo assim a microbiota intestinal. Assim, intervenções dietéticas são boas opções de tratamento. (Li, et al., 2015).

Terapias nutricionais que envolvam nutrição enteral (NE), modificação de carboidratos e fibras alimentares vêm sendo discutidas e prescritas no meio pediátrico como propostas terapêuticas e preventivas para crises de atividade inflamatória 
na Doença de Crohn (DC) e retocolite ulcerativa (RCU). (Lane, et al., 2017).

A nutrição enteral exclusiva é indicada na fase aguda da doença e a nutrição enteral suplementar é indicada para manter a doença em remissão e para alcançar o aumento de peso (O'sullivan, et al., 2006).

A nutrição parenteral é um método alternativo para ingestão enteral de nutrientes em pacientes com doenças inflamatórias intestinais, é usado principalmente em pacientes com contraindicações ou intolerância a nutrição enteral e especialmente quando a desnutrição grave. A nutrição parenteral total pode ser usada pode ser usada durante a fase inflamatória aguda (obstrução, megacólon tóxico e fístulas ativas) no período pré-operatório e em pacientes com síndrome do intestino curto devido a ressecções intestinais extensas anteriores (Alastair, et al., 2011).

\section{Metodologia}

O estudo tem uma abordagem qualitativa, pois fará uma análise que respeite os resultados colhidos nas investigações e ao mesmo tempo traçará paralelos entre as referências bibliográficas oriundas de fontes teóricas de autores respeitados que discutem o assunto. $\mathrm{O}$ pesquisador qualitativo pauta seus estudos na interpretação do mundo real, preocupando-se com o caráter hermenêutico na tarefa de pesquisar sobre a experiência vivida dos seres humanos.

Para Prus apud Moreira (2002), a tarefa de "dupla hermenêutica" justifica-se pelo fato de os investigadores lidarem com a interpretação de entidades que, por sua vez, interpretam o mundo que as rodeiam. $\mathrm{O}$ autor ainda nos elucida que os objetos de estudo das ciências humanas e sociais são as pessoas e suas atividades, considerando-os "não apenas agentes interpretativos de seus mundos, mas também compartilham suas interpretações à medida que interagem com outros e refletem sobre suas experiências no curso de suas atividades cotidianas".

A coleta de dados será por meio de revisão bibliográfica para levantamentos secundários nos bancos de dados online PUBMED, SCIELO e LILACS entre 2010 e 2020. Essas publicações são consideradas como destaque, sendo de extrema importância para esse estudo. Em relação à pesquisa foi realizada uma revisão descritiva exploratória, fazendo assim o pesquisador adaptar-se com o que está sendo investigado durante a pesquisa.

Os dados serão analisados de acordo com as normas científicas, dessa forma faz-se necessário que seja obedecido todos os protocolos exigidos pelos métodos de pesquisa da área da nutrição. Esta análise levará em consideração materiais que tratem das doenças inflamatórias intestinais, onde os artigos são referentes a população adulta no ano de 2010 a 2020. Já os critérios de exclusão foram artigos voltados a crianças e publicados nos anos anteriores a 2010. Dessa forma a coleta dos respectivos dados se pautará em descobrir as características nutricionais das doenças inflamatórias intestinais, visando compreender qual o prognóstico nos diferentes tipos de doenças no estado nutricional e qual a melhor terapia e tratamento das mesmas.

\section{Resultados e Discussão}

Segundo Gomes \& Santos \& Ferreira (2010) a Doença de Crohn e a Retocolite Ulcerativa se comportam de maneira semelhante, por isso estão agrupadas na categoria de doenças inflamatórias intestinais. O que difere elas são que, na doença de Crohn todas as partes estão envolvidas, podendo conter segmentos de intestino saudável e intestino doente, já a Retocolite Ulcerativa afeta apenas a mucosa (camada mais superficial do colón) de modo continuo.

Segundo Beyer (2010) a Doença de Crohn tem apresentação bastante característica, seu acometimento se dá em forma segmentar, é caracterizado por possuir segmentos saudáveis e inflamados. Com isso as complicações da doença incluem fibrose intestinal, estenoses e formação de fistulas (Iskandar, et al., 2012).

Fatores de risco como alimentação, tabagismo, utilização de anti-inflamatórios não esteroides, infecções intestinais e o 
uso de contraceptivos orais estão relacionados a doença. Existem alguns produtos alimentares quem podem conduzir a uma eventual inflamação intestinal. Alterações na composição da flora entérica ou alterações na permeabilidade do intestino estão envolvidos ao efeito antigénico direto. (Hou, et al., 2011).

As características das DII, estão ligadas a fatores ambientais, genético, a microbiota intestinal, e o sistema imunológico, estão envolvidos e funcionalmente integrados na etiologia da reação inflamatória crônica. A Retocolite Ulcerativa pode resultar de uma resposta imune exagerada da mucosa do cólon a antígenos luminais, que podem ser microbianos, em indivíduos com predisposição genética. (Dani, et al., 2011).

Dietas ricas em bebidas à base de cola, açúcar refinado, chocolate, com poucas fibras e rica em gorduras estão relacionadas à Retocolite Ulcerativa, com isso, o consumo desses produtos pode representar um possível fator de risco. Estudos recentes mostram que a falta de leite materno pode estar associada ao desenvolvimento da Retocolite Ulcerativa. Mesmo com esses resultados, a associação da dieta ainda não está clara à etiopatogênese da Retocolite Ulcerativa, com isso é necessário mais estudo nesta área (Dani, et al., 2011).

\subsection{Doença de Crohn}

A doença de Crohn é uma doença inflamatória crônica do trato gastrointestinal com sintomas que evoluem de forma recorrente e remitente é uma doença progressiva que causa danos ao intestino e incapacidade. Todos os segmentos do trato gastrointestinal podem ser afetados, principalmente o íleo terminal e o colón. A inflamação é segmentar assimétrica e transmural. Os pacientes apresentam um fenótipo inflamatório no momento do diagnostico, mas com o tempo podem ter complicações com estenoses, fístulas, ou abcessos, o que podem resultar em cirurgias. (Thia, et al., 2010).

A doença se inicia geralmente entre segunda e quarta década de vida, com um pico menor que é descrito entre 50 e 60 anos. Tem crescido de forma constante na maioria das regiões do mundo. Sua incidência e prevalência são maiores em países desenvolvidos do que em países em desenvolvimento e em áreas urbanas do que em áreas rurais. (Molodecky, et al., 2012).

A doença de Crohn pode estar relacionada com a interação entre a suscetibilidade genética, fatores ambientais e a microflora intestinal, o que resulta em uma resposta imune da mucosa anormal e comprometimento da função da barreira epitelial. Aproximadamente 12\% dos pacientes têm história familiar de doença de Crohn. (Moller, et al., 2015).

O tabagismo está associado ao aumento de duas vezes no risco para doença de Crohn, na infância alguns antibióticos podem aumentar o risco de doença de Crohn (Ungaro, et al., 2014). A redução na fibra dietética e um aumento na ingestão de gordura saturada também foram associados a um risco aumentado da doença (Ananthakrishnan, et al., 2014).

\subsection{Retocolite ulcerativa}

A retocolite ulcerativa é um tipo de doença inflamatória intestinal, caracterizada por inflamação continua e difusa é limitada a mucosa do cólon e se estende proximalmente a partir do reto. Se desenvolve mais frequentemente na segunda ou terceira década de vida. Seus principais sintomas são diarreia com sangue, dor abdominal, urgência fecal e/ou tenesmo. (Danese, et al., 2011).

A incidência de retocolite ulcerativa está relacionada a fatores ambientais, um dos principais fatores é o ex-tabagismo, os fumantes ativos estão menos expostos a desenvolver a retocolite ulcerativa quando comparados a antigos fumantes e não fumantes, além disso podem ter a doença em forma leve. (Ananthakrishnan, et al., 2015).

A apendicectomia pode ter um efeito protetor contra o desenvolvimento de retocolite ulcerativa principalmente quando feita em pacientes jovens. (Sahami, et al., 2016).

Os fatores de risco podem estar relacionados a perturbações no intestino barreiras mucosas e perturbações resultantes da microbiota intestinal. (Danese, et al., 2011). As infecções entéricas foram associadas ao desenvolvimento das doenças 
inflamatórias intestinais, mais especificamente Salmonella e Campylobacter.

\subsection{Características nutricionais das doenças inflamatórias}

Segundo Kostic \& Xavier \& Gevers (2014) a dieta tradicional das gerações anteriores, quando a prevalência de Doenças inflamatórias intestinais era consideravelmente mais baixa, é consideravelmente diferente da dieta atual. A dieta ocidental é caracterizada pelo grande consumo de açúcar refinado, gorduras poli-insaturadas ômega-6 e fast-food, e baixo consumo de frutas, vegetais e fibras.

Antigamente os alimentos produzidos localmente, eram consumidos logo após a colheita, nos dias atuais a alimentação é processada, modificada, armazenada e transportada por grandes distancias até chegar ao consumidor final. Acredita-se que essa mudança na alimentação pode aumentar essas citocinas pró-inflamatórias que pode causar inflamação crônica de baixo grau no intestino. (Eeckhaut, et al., 2014).

O consumo de alimentos pró-inflamatórios é um fator de risco importante, para o desenvolvimento de Retocolite Ulcerativa. Enriquecer a dieta com alimentos anti-inflamatórios, por exemplo vegetais ricos em fibras e fitoquímicos, diminuir a ingestão de fatores pró inflamatórios, como alimentos fritos ou processados, ricos em ácidos graxos pode ser uma estratégia potencial para reduzir o risco de Retocolite Ulcerativa. (Derwa, et al., 2017).

As dietas orientais são baseadas em carboidratos derivados de plantas, vegetais, arroz e frutas o que faz com que a sua microbiota tem uma maior prevalência de Prevotella spp, e a população ocidental tem maior prevalência de bacteroides spp. Proteínas e gorduras de fontes animais, estão associadas a um aumento de bacteroides spp. por outro lado, os carboidratos simples e fibras estão principalmente associados a um aumento de Prevotella ssp. (Molodecky, et al., 2012).

A microbiota intestinal pode ser destruída por ditas ricas em gordura ou açúcar o que leva à disbiose e aumento da produção de endotoxinas (Varela, et al., 2013). Com a disbiose, a mucosa intestinal é modificada, tornando mais fina e mais permeável a patógenos e antígenos causando o estabelecimento de uma inflamação de baixo grau, mas recorrente (Wills, et al., 2014). A dieta rica em fibras e vegetais, diminuem o pH intestinal, evitando o crescimento de bactérias patogênicas, como por exemplo, as cepas de E. coli e Enterobacteriaceae (Gearry, et al., 2016).

A qualidade dos alimentos e a dieta influencia a composição da microbiota e representa uma fonte de antígenos luminais, foi observado que a dieta de estilo ocidental pode ser um gatilho para Retocolite Ulcerativa e Doença de Crohn (Kelder, et al., 2014).

\subsection{Estado nutricional de pacientes com doenças inflamatórias intestinais}

A desnutrição proteico-energética, é comum entre pacientes com Doenças Inflamatórias Intestinais, tem composição corporal alterada e deficiência de micronutrientes. A desnutrição está relacionada a resultados ruins em pacientes com essa patologia. (Sandhu, et al., 2015). O principal contribuinte para o baixo peso em adultos com doença de Crohn é a má absorção. (Vitek, et al., 2015). A enteropatia proteica também pode contribuir com a perda de nutrientes e são características de um intestino rompido e permeável, a atividade da doença é paralela à perda de proteína gastrointestinal. (Lee, et al., 2015).

Foi realizado um estudo no Estados Unidos que mostrou, uma prevalência de obesidade em pacientes com Doenças Inflamatórias Intestinais, esse dado reflete o índice de obesidade na população em geral. Um outro dado importante é que pacientes obesos, tem melhores resultados clínicos, ou seja, a obesidade é um marcador de doença menos grave, nas Doenças Inflamatórias Intestinais. Essa patologia pode ter um diagnóstico precoce em pacientes obesos. (Flores, et al., 2015).

\subsubsection{Composição corporal}

Para o estudo do estado nutricional, o peso e IMC são índices muito superficiais, por isso outras medidas corporais 
são utilizadas. Pacientes com doenças inflamatórias intestinais tem a massa corporal magra menor que pessoas saudáveis (Bryat, et al., 2015).

Um fator considerado patogênico para doença inflamatória intestinal é a distribuição de gordura, especialmente se estiver localizada em torno do lúmen do intestino. Por ser considerada patognomônica. (Fink, et al., 2012). Um outro fator importante para a etiologia da doença é que o tecido adiposo secreta as adipocinas, que são pró-inflamatórias e moduladoras do sistema imunológico. (Ponemone, et al., 2010).

\subsubsection{Massa Óssea}

Um fator de risco para diminuição na densidade óssea é quando a doença inflamatória intestinal tem um início precoce, antes dos 30 anos de idade (Kim, et al., 2013). Porém quando tem início depois de 50 anos também é considerada um fator de risco para osteopenia e osteoporose (Miheller, et al., 2013). Em um estudo italiano foi observado que, pacientes do sexo masculino e com 30 anos de idade foram diagnosticados com doenças inflamatórias intestinais, osteoporose e osteopenia foi maior do que em mulheres. (Adriani, et al., 2014)

Porém um estudo semelhante realizado nos Estados Unidos não identificou a influência da idade, IMC ou localização da doença na densidade óssea (Abrahan, et al., 2014). No entanto um estudo americano mostrou a relação positiva entre a densidade mineral óssea e o IMC (Gupta, et al., 2014). Um outro estudo da Eslováquia mostrou que os pacientes com DC com localização ileica / ileocólica e história de proctocolectomia / colectomia total tinham um risco maior de desenvolver osteoporose do que outros pacientes com DII (Miznerova, et al., 2014).

A má absorção do cálcio e da vitamina D estão relacionados a perda da matriz óssea uso de esteroides sistemático de longo prazo, também estão relacionados a essa perda. (Targownik, et al., 2014). Melhores resultados da densidade mineral óssea estão relacionados a terapia biológica. (Pichler, et al., 2014). Nas doenças inflamatórias intestinal a densidade mineral óssea está relacionada com fatores genético (Van et al 2014) mediadores inflamatórios, como as adipocinas, interferem no equilíbrio entre osteoblastos e osteoclastos (Terzoudis, et al., 2014).

\section{Terapia nutricional para doenças inflamatórias intestinais}

\subsection{Nutrição oral}

\subsubsection{Dieta específica de carboidratos}

A dieta especifica de carboidratos recomenda a exclusão de carboidratos complexos, dando preferência a monossacarídeos que é mais fácil de digerir e sua absorção também é mais fácil. Com isso fica menor a concentração de carboidratos diminuindo a fermentação bacteriana e lesão intestinal. É uma dieta que permite carne não processadas, aves, ovos, peixes, frutas, vegetais, todas as gorduras e óleos. (Suskind, et al., 2014).

Os dados iniciais são promissores, porém são necessários mais estudos. A restrição da dieta pode diminuir a adesão e satisfação do paciente com terapia de longo prazo, embora a dieta possa ser liberada após a remissão da doença.

\subsubsection{Dieta restrita em carboidratos fermentáveis}

Os carboidratos de cadeia curta são mal absorvidos no intestino delgado, causando um aumento de secreção de água, fermentação bacteriana, e produção de gás em excesso. Esses carboidratos são: oligossacarídeos fermentáveis, dissacarídeos, monossacarídeos e polióis. Esses processos levam a distensão luminal, desconforto abdominal, disbiose, lesão da mucosa e hábitos intestinais alterados. (Ong, et al., 2010).

Nessa dieta a redução de carboidratos fermentáveis por vários dias, depois é feita a reintrodução dos alimentos, 
utilizando um diário alimentar para identificar individualmente alguns gatilhos.

Os estudos que utilizam essa dieta, tem focado na melhoria dos sintomas e não no controle da inflamação o que limita a capacidade de saber se essa dieta serve como tratamento das doenças inflamatórias intestinais.

\subsubsection{Dieta anti-inflamatória}

Inicialmente a dieta se concentrava em equilibrar as porções de macronutrientes para melhorar os níveis de cortisol e insulina. Outras dietas que surgiram focaram em evitar macronutrientes com propriedades pró-inflamatórias, e consumir aqueles com propriedades anti-inflamatórias. Em geral as dietas anti-inflamatórias incluem o aumento de frutas e vegetais, proteínas vegetais, algumas proteínas animais magras, peixes gordurosos, fibras e certas ervas e especiarias, grãos inteiros podem ser consumidos com moderação, o azeite pode ser utilizado como fonte de gordura, diminuição de carboidratos refinados. Os alimentos devem ser consumidos por qualidade e não quantidade. (Ricker, et al., 2017).

A dieta anti-inflamatória para doenças inflamatórias intestinais é uma derivação da dieta específica de carboidratos, seguindo as mesmas suposições que o consumo de certos carboidratos podem levar a disbisose, lesão intestinal e aumento de permeabilidade intestinal. Os componentes básicos são: restrição de carboidratos, uso de prebiótico e probiótico, redução dos ácidos graxos totais e saturados, redução da textura dos alimentos e a fibra intacta para melhor absorção. (Gunasekeer, et al., 2016).

\subsubsection{Dieta rica em fibras}

As fibras dietéticas são polímeros de carboidratos não hidrolisados por enzimas endógenas no intestino delgado. As fibras indigestíveis são fermentadas pelas bactérias intestinais, o que leva a vários benefícios posteriores, regulando a absorção de fluido e eletrólito, melhora a barreira da mucosa, tem alterações favoráveis na microbiota intestinal. E propriedades antiinflamatórias. Com esses benefícios tem aumentado o consumo de fibras em pacientes com doenças inflamatórias intestinais. (Jhones, et al., 2014).

\subsubsection{Dieta de baixo resíduo}

Diferente da dieta rica em fibras, a dieta de baixo resíduo é concentrada na redução de nutrientes mal digeridos (ou seja, fibra) minimizando a saída das fezes. Esse tipo de dieta é usado frequentemente em pacientes com doença de Crohn, com doença estenosante e pacientes submetidos a cirurgia abdominal. É uma dieta que inclui macronutrientes de fácil absorção para reduzir a exposição a jusante dos antígenos alimentares aos intestinos. Alguns tipos de proteínas complexas são considerados alergênicos para indivíduos suscetíveis. (Hanai, et al., 2012).

\subsection{Dieta Enteral e Parenteral}

\subsubsection{Dieta Enteral}

A alimentação enteral é usada na terapia nutricional das doenças inflamatórias intestinais a mais de 40 anos. Teve início na década de 70, após os efeitos terapêuticos primários de uma dieta elementar em pacientes com doença de Chron, foram realizados vários estudos sobre nutrição enteral, foi observado que o efeito terapêutico era diferente, dependendo do tipo de doença inflamatória intestinal, a idade do paciente, suas apresentações clinicas e seu tratamento concomitante. (Wall, et al., 2013)

A nutrição enteral pode atingir até $100 \%$ das necessidades nutricionais de um paciente utilizando fórmula nutricional liquida por via oral ou através de um tubo próprio para alimentação. Essa dieta geralmente é fornecida de 6 a 8 semanas, em seguida pode se reintroduzida a dieta normal. (Whitten, et al., 2012). 
A composição da fórmula enteral, a via de administração, fazem com que os protocolos de nutrição enteral sejam diferentes. Os tipos de fórmulas usadas na nutrição enteral dependem do grau de hidrolise da proteína. Fórmulas poliméricas são utilizadas para imitar a dieta geral com proteínas não hidrolisadas, carboidratos e gordura. A principal proteína é a caseína, a maltodextrina de milho é utilizada como fonte de carboidratos, e a fonte de gordura é a canola ou a soja. As fórmulas semielementares e elementares são utilizadas em pacientes com má absorção, para que os nutrientes sejam parcialmente (que é o caso das fórmulas semi-elementares), ou totalmente hidrolisados (que é o caso das fórmulas elementares). Nas fórmulas semielementares a proteína está na forma de dipeptídeos ou tripeptídeos, já nas fórmulas elementares os aminoácidos estão em forma livre, os carboidratos são fornecidos como amido de milho hidrolisado, maltodextrina ou frutose. (Brown, et al., 2015).

Na nutrição enteral, não há nenhum componente especifico que possa explicar o efeito curativo desta terapia, mas alguns componentes como glutamina, arginina e ácidos graxos poli-insaturados, mostram ter propriedades benéficas para pacientes com doenças inflamatórias intestinais. (Alhagamhmad, et al., 2012).

\subsubsection{Dieta Parenteral}

Algumas pesquisas sugerem que 20\% a $85 \%$ dos pacientes que tem doenças inflamatórias intestinais estão desnutridos ou abaixo do peso. (Semrad, et al., 2012). Pacientes com doença de Crohn tem mais desnutrição, que pacientes com retocolite ulcerativa. Os motivos da desnutrição em pacientes com doenças inflamatórias intestinais estão relacionados a condição do intestino delgado no peso corporal do paciente (Guagnozzi, et al., 2012).

A nutrição parenteral pode trazer benefícios a pacientes com doenças inflamatórias intestinais é utilizado como alternativa para ingestão enteral de proteínas, gorduras, vitaminas e minerais. É utilizada em pacientes que tenham contra indicação ou intolerância a nutrição enteral. E principalmente aqueles que tenham sintomas de desnutrição grave. Também é usada durante a fase inflamatória aguda (obstrução, megacólon toxico e fistulas ativas). (Mihai, et al., 2013).

A nutrição parenteral é usada principalmente no período pré-operatório em pacientes com desnutrição grave para restituição ou melhora de sua condição metabólica. Alguns benefícios são melhora na sua cicatrização de feridas, prevenção de complicações pós-operatórias e perda de peso. (Papic, et al., 2013).

Os riscos de complicações relacionadas a distúrbios metabólicos, insuficiência hepática, estão aumentados com o uso de nutrição parenteral prolongado o que pode ocasionar a qualidade de vida do paciente. A nutrição enteral é o tipo de alimentação mais recomendada se o intestino poder ser usado na fase de indução e remissão do tratamento. (Guagnozzid, et al., 2012).

\section{Conclusão}

Portanto, o presente trabalho abordou de forma consistente os diversos benefícios da terapia nutricional no que diz respeito ao tratamento de doenças inflamatórias intestinais. Foi observado que para que se tenha uma habilitação considerável neste aspecto patológico, faz-se necessário a intervenção nutricional da melhor forma possível. Evidenciando que os conceitos da nutrição são cruciais para o tratamento nessas circunstâncias.

Para esta tarefa é importante que se tenha o conhecimento das principais características nutricionais concernentes ao tratamento das doenças inflamatórias intestinais, sendo que tal estudo mostrará os caminhos a serem tomados para beneficiar o paciente com o método mais eficaz. Partindo deste conhecimento prévio, serão estabelecidas estratégias nutricionais mais formidáveis mediante uma gama maior de aprendizado.

É crucial que seja feita uma análise com o maior rigor cientifico possível dos métodos nutricionais enteral e parenteral e dessa forma definir quais são os modelos mais adequados para o tratamento das doenças inflamatórias intestinais, para que 
em seguida sejam tomadas medidas relevantes para o tratamento das pessoas que necessitam desses mecanismos que viabilizam benefícios na saúde dos pacientes, estabelecendo com essa terapia um padrão que introduza a nutrição como uma aliada da medicina no combate a esse tipo de doença que afeta uma parcela expressiva da população, deste modo faz-se necessário continuar buscando terapias nutricionais que ajudem na recuperação dos pacientes que tem doenças inflamatórias intestinais, principalmente a doença de crohn e retoculite ulcerativa.

\section{Referências}

Abraham, B. P.; Prasad, P.; \& Malaty, H. M. (2014). Vitamin D deficiency and corticosteroid use are risk factors for low bone mineral density in inflammatory bowel disease patients. Dig Dis Sci . 59, 1878-84 [PMID: 24619280 DOI: 10.1007/s10620-014-3102-x].

Adriani, A.; Pantaleo, S.; Luchino, M.; Ribaldonei, D.G.; Reggiani, S.; Sapone, N.; Sguazzini, C.; Isaia, G.; Pellicano, R.; \& Astegiano, M. (2014) Osteopenia and osteoporosis in patients with new diagnosis of inflammatory bowel disease. Panminerva Med. 56, 145-149 [PMID: 24994578].

Alastair, F.; Emma, G.; \& Emma, P. (2011). Nutrition in inflammatory bowel disease. JPEN J Parenter Enteral Nutr. 35, 571-580 [PMID: 21825089 DOI: $10.1177 / 0148607111413599]$.

Alhamhmad, M. H .; Day, A. S.; Lemberg, D.A. \& Leach, S. T. (2012). An update of the role of nutritional therapy in the management of Crohn's disease. $J$ Gastroenterol , 47: 872-882 [PMID: 22699323 DOI: 10.1007/s00535-012-0617-9] .

Ananthakrihnan, A. N. (2015). Epidemiology and risk factors for IBD. Nat Ver Gastroenterol Hepatol. 12, $205-17$.

Ananthakrishnan, A. N.; Khalili, H.; \& Konijeti, G. G.; et al. (2014) Ingestão de gordura alimentar a longo prazo e risco de colite ulcerosa e doença de Crohn. Intestino. 63, 776-84.

Beyer, P.L.(2010). Tratamento médico nutricional para doenças do trato gastrointestinal inferior. In: MAHN, L. K.; ESCOTT-STUMP, S. Alimentos, Nutrição e Dietoterapia. 12. ed. Rio de Janeiro: Elsevier. 689-695.

Brown, B.; Roehl, K.; \& Betz, M. (2015). Enteral nutrition formula selection: current evidence and implications for practice. Nutr Clin Pract. 30, 72-85 [PMID: 25516537 DOI: 10.1177/0884533614561 791

Bryat, R. V.; Ooi, S.; Schutz, C.G.; Goess, C.; Grafton, R.; Hughes, J.; Lim, A.; Bartholomwusz, F.D.; \& Andrews, J.M.(2015). Low muscle mass and \begin{tabular}{l} 
sarcopenia: common and predictive of osteopenia in inflammatory bowel disease. Aliment $\quad$ Pharmacol Ther. $41,895-906 \quad$ [PMID: \\
\hline
\end{tabular} 25753216 DOI: 10.1111/apt.13156] .

Danese, S.; \& FiocchiI, C. Ulcerative colitis. (2011). N Engl J Med. 365, (18):17131725

Dani, R.; Passos.; \& Friche, M. C. F. (2011). Gastroenterologia essencial. (4. ed.) Guanabara. Koogan.

Derway.; Gracie, D. J.; \& Hamlin, P. J. (2017) Ford, A C Revisão sistemática com metaanálise: A eficácia dos probióticos na doença inflamatória intestinal. Aliment. Pharmacol. Ther. 46, 389-400.

De Souza, H. S \& Fiocchi, C. (2016) Imunopatogênese do IBD: estado da arte atual. Nat. Rev. Gastroenterol. Hepatol. 13, 13-27 . Molodecky, NA et al. Crescente.

Eeckhat, V.; Ducatelle, R.; Sas, B.; Vermeire, S.; \& Immerssel, V. F. (2014) Progresso em direção aos farmabióticos produtores de butirato: cápsula de Butyricoccus pullicaecorum e eficácia em modelos de TNBS em comparação com a terapêutica. Gut. $63,367$.

Fink, C.; Karagiannide, I.; Bakirtzi, K.; \& Pothoulakis, C. (2012). Adipose tissue and inflammatory bowel disease pathogenesis. Inflamm Bowel Dis. 18, 15501557 [PMID: 22407798 DOI: 10.1002/ibd.22893]

Flores, A.; Burstein, E.; Cipher, D.J.; \& Feangis, L.A.(2015). Obesidade na doença inflamatória intestinal: um marcador de doença menos grave. Dig Dis Sci. 60, 2436-2445 [PMID: 25799938].

Gearry, R. B. (2016). IBD e meio ambiente: Existem diferenças entre leste e oeste. Digerir. Dis. 34, 84-89.

Gomes, G.C. ; Santos, P. M. F.; \& Ferreira, S. L. V. (2010). Vivências de Pessoas Ostomizadas com Doença de Crohn. Coimbra: Revista Referência.12, 1934.

Guagnozzi, D.; Gonzalez, C. S.; Oliveira, A.; \& Lucendo, A.J. (2012). Nutritional treatment in inflammatory bowel disease. An update. Rev Esp Enferm. Dig. 104, 479-488 [PMID: 23130856].

Gunasekeer, V.; Mendall, M.A.; \& Chan, D. (2016) Tratamento da doença de Crohn com uma dieta de exclusão guiada por IgG4: um ensaio clinico randomizado. Dig Dis Sci. 61 (4), $1148-57$.

Gupta, S.; Wu, X.; Moore, T.; \& Shen, B. (2014). Frequency, risk factors, and adverse sequelae of bone loss in patients with ostomy for inflammatory bowel diseases. Inflamm Bowel Dis. 20, 259-264 [PMID: 24378598 DOI: 10.1097/01.MIB.0000439065.92211.d3].

Hou, J.K.; Abraham, B.; \& El-Serag, H. (2011). Dietary intake and risk of developing inflammatory bowel disease: a systematic review of the literature. Am J Gastroenterol. 106, 563-73. https://doi.org/10.1038/ajg.2011.44. 
Hou, J. K.; Abraham, B.; \& El-seragL, H. (2011). Dietary intake and risk of developing inflammatory bowel disease: a systematic review of the literature. American Journal Gastroenterology. 106, 563-573.

Iskandar, H. N.; \& Ciorba, M. A. (2012). Biomarkers in inflammatory bowel disease: current practices and recent advances. Translational Research. v.159 , $313-325$.

Jones, J. M. (2014). As definições de fibras dietéticas alinhadas com CODEX ajudam a preencher a” lacuna de fibra”. Nutr J. 13-34.

Kelder, T.; Stroeve, J.H.; Bijlsma, S.; \& Radonjic, M.; Roeselers, G. (2014). Análise de rede de correlação revela relações entre mudanças induzidas por dieta na microbiota intestinal humana e saúde metabólica. Nutr Diabetes. 4-122.

Kim, H. J.; Hong, S. J.; Jeon, Y. W.; Han, J. P.; Han, S.H.; Kang, J. H.; Tae, J. W.; Lim, H. S.; Kim, H. K.; Ko, B. M.; \& Lee, M. S. (2013). The early onset of disease may be a risk factor for decreased bone mineral density in patients with inflammatory bowel disease. Clin Endosc. 46, 71-76 [PMID: 23423611 DOI: $10.5946 / \mathrm{ce} .2013 .46 .1 .71]$.

Kostic, A D.; Xavier, R .J.; \& Gevers, D. (2014). O microbioma na doença inflamatória intestinal: Status atual e o futuro à frente. Gastroenterology. 146, $1489-1499$.

Lane, E. R,; Lee, D,; \& Suskind, D L. (2017). Dietary therapies in pediatric inflammatory bowel disease: an evolving inflammatory bowel disease paradigm. Gastroenterol Clin North Am. 46, 731-44. https://doi.org/10.1016/j.gtc.2017.08.012.

Lee, S .H. (2015). Regulação da permeabilidade intestinal por tight junction: implicações nas doenças inflamatórias intestinais. Intest Res. 13, 11-18 [PMID: 25691839 DOI: 10.5217 / ir.2015.13.1.11] MIB.0000000000000193].

Li, J.; Butcher, J.; Mack, D.;\& Stintzi, B. A. (2015). Functional impacts of the intestinal microbiome in the pathogenesis of inflammatory bowel disease. Inflamm Bowel Dis. 21, 139-53. https:// doi.org/10.1097/MIB.0000000000000215.

Mihai, C.; Prelipcean, C. C.; Pintilie, I.; Nedelciuc, O,; Jigaranu, A.O,; Dranga, M,; \& Mihai, B.(2013). Nutrição em doenças inflamatórias intestinais. Rev Med Chir Soc Med Nat Iasi. 117, 662-669 [PMID: 24502032].

Miheller, P.; Gesztes, W.; \& Lakatos, P. L. (2013). Manipulating bone disease in inflammatory bowel disease patients. Ann Gastroenterol. 26, 296-303 [PMID: 24714303].

Miznerova, E.; Hlavatyl, T.; Koller, T.; Toht, J.; Holociova, K.; Huorka, M.; Killiger, Z.; \& Payer, J. (2013). The prevalence and risk factors for osteoporosis in patients with inflammatory bowel disease. Bratisl Lek Listy. 114, 439-445 [PMID: 23944617].

Moller, F.T.; Andersen, V.; Wohlfahrt, \& J.; Jess, T. (2015). Risco familiar de doença inflamatória intestinal: um estudo de coorte de base populacional 19772011. Am J Gastroenterol . 110, 564-71.

Molodecky, N.A.; Rabi, D. M.; Ghali, W.A.; Ferris, M.; Chernof, G.; Benchimol, E. I; Panaccione, R.; Ghosh, S.; \& Barkema, H. W.; et al. (2012). Aumento da incidência e prevalência das doenças inflamatórias intestinais com o tempo, com base em revisão sistemática. Gastroenterology. 142, 46-54.

Moreira, D. A. (2002). O método fenomenológico na pesquisa. São Paulo: Pioneira Thomso.

Hanai, H.; Ilda, T.; \& Takeuchi, K. (2012). Terapia nutricional versus 6- mercaptopurina como terapia de manutenção em pacientes com doença deCrohn. DigLiver Dis. 44 (8), 649-54.

Ong, D. K.; Mitchel, S. B.; \& Barret, J. S.; et al. (2010). Manipulação de carboidratos de cadeia curta da dieta hidratos altera o padrao de produção de gas e gêneses dos sintomas na síndrome do intestino irritável. J Gastroenterol Hepatol. 25(8), 1366-1373.

O'sullivan, M.; \& O'morain, C. (2006). Nutrição na doença inflamatória do intestino. Best Pract Res Clin Gastroenterol. 20, 561-573 [PMID: 16782529 DOI: $10.1016 /$ j.bpg.2006.03.001.

Papi, C.; Fasci, S. F.; Rogai, F.; Settesoldi, A.; Margagnoni, G.; \& Annese, V. (2013). Mucosal healing in inflammatory bowel disease: treatment efficacy and predictive factors. Dig Liver Dis. 45, 978-985 [PMID: 24018244 DOI:

10.1016/j.dld.2013.07.006].

PichlerI, J.; Hanslik, A.; Huber, W. D.; Aufricht, C.; \& Bidmon, F. B. (2014) Paediatric patients with inflammatory bowel disease who received infliximab experienced improved growth and bone health. Acta Paediatr. 103,69-75 [PMID: 24237381 DOI: 10.1111/apa.12448].

Ponemone, V.; Keshavarzia, A.; Brand, M. I.; Saclarides, T.; Abcarian, H.; Cabay, R J.; Fletcher, E.; Larsen, B.; Durstine, L. J.; Fantuzzi, G.; \& Fayad, R. (2010). Apoptosis and inflammation: role of adipokines in inflammatory bowel disease. Clin Transl Gastroenterol 1: e1 [PMID: 23238652 DOI: 10.1038/ctg.2010.1].

Ricker, M. A.; \& Haas, W. C. (2017). Dieta antinflamatoria na prática clínica : uma revisão . Nutr Clin Pract . 32 (3), 318 - 25.

Sahami, S.; Kooij, I. A.; Meijer, S. L.; Van, G.R.; Buskens, C .J.; \& Te, A. A. (2016). A ligação entre o apêndice e a colite ulcerosa: relevância clínica e potenciais mecanismos imunológicos. Am J Gastroenterol. 111, 163-69.

Sandhu, A.; Moasli, M.; Yan, B.; Wu, T.; Gregor, J.; Chande, N.; Poich, T.; Beaton, M.; \& Rahman, A. (2015). AutoScreening para risco de desnutrição em pacientes ambulatoriais com doença inflamatória intestinal usando a ferramenta universal de triagem de desnutrição (MUST). JPEN J Parenter Nutr Enteral. Epub ahead of print [PMID: 25632031].

Semrad, C E. (2012). Use of parenteral nutrition in patients with inflammatory bowel disease. Gastroenterol Hepatol. 8, 393-395 [PMID: 22933876]. 
Research, Society and Development, v. 10, n. 7, e11410716660, 2021

(CC BY 4.0) | ISSN 2525-3409 | DOI: http://dx.doi.org/10.33448/rsd-v10i7.16600

Suskind, D. L.; Wahbeh, G.; \& Gregory, N. (2014). Terapia nutricional em Crohn pediátrico doença: a dieta de carboidratos específicos. J Pediatr Gastroenterol Nutr. 58, (1) 87-91.

Targownik, L. E.; Bernstein, C .N.; \& Leslie, W. D. (2014). Risk factors and management of osteoporosis in inflammatory bowel disease. Curr Opin Gastroenterol. 30, 168-174 [PMID: 24419292 DOI: 10.1097/MOG.0000000000000037]

Terzoudis, S.; Zavos, C.; Koutrobakis, I.E. The bone and fat connection in inflammatory bowel diseases. Inflamm Bowel Dis 20, $2207-2217$ [PMID: 24983981 DOI: 10.1097/ MIB.0000000000000111].

Thia, K. T.; Sandborn, W. J.; Harmsen, W. S.; Zinsmeister, A.R.; \& Loftus, E.V. (2010). Fatores de risco associados à progressão para complicações intestinais da doença de Crohn em uma coorte de base Gastroenterologia populacional. 139, 1147-55.

Ungaro, R.; Bernstein, C.N.; \& Gearry, R.; et al. Antibióticos associados ao risco aumentado de doença de Crohn de início recente, mas não colite ulcerosa: uma meta-análise. Am J Gastroenterol .109, 1728-38.

Van, S. S.; Janse, M.; Karjalainen, J.; Fehrmann, R.; Franke, L.; Fu, J.; \& Weersmar, K. (2014). Extra intestinal manifestations and complications in inflammatory bowel disease: from shared genetics to shared biological pathways. Inflamm Bowel Dis. 20, 987-994 [PMID: 24739630 DOI: 10.1097/ MIB.0000000000000032].

Varela, E.; ManichanhA, C.; Gallart, M.; Torrejón, A.; Borruel, N.; Casselas, F.; Guaner, F.; Antolin, M. (2013). Colonization by Faecalibacterium prausnitzii e manutenção da remissão clínica em pacientes com colite ulcerosa. Aliment. Pharmacol. Ther. 38, 151-161.

Vítek, L.(2015). Má absorção de ácido biliar na doença inflamatória intestinal. Inflamação intestinal 21: 476-483 [PMID: 25248001 DOI: 10.1097.

Wall, C. L.; Day, A.S.; \& Gearry, R. B. (2013). Use of exclusive enteral nutrition in adults with Crohn's disease: a review. World J Gastroenterol. 19, 76527660 [PMID: 24282355 DOI: 10.3748/wjg.v19.i43.7652] .

Whitten, K. E.; Rogers, P.; Ooi, C. Y.; \& DayY, A. S.(2012). International survey of enteral nutrition protocols used in children with Crohn's disease. J Dig Dis 13, 107-112 [PMID: 22257479 DOI: 10.1111/ j.1751-2980.2011.00558.x].

Willis, E. S.; Jonkers, Dmae.; Savelkoul, P. H.; Masclee, A. A.; Pierik, M.J.; \& Penders, J. (2014). Composição microbiana fecal da colite ulcerosa e pacientes com doença de Crohn em remissão e exacerbação subsequente. PLoS ONE . 9, 90-981. 